\title{
The role of combined SNV and CNV burden in patients with distal symmetric polyneuropathy
}

\author{
Davut Pehlivan, MD ${ }^{1,2}$, Christine R. Beck, PhD1, Yuji Okamoto, MD, PhD1, \\ Tamar Harel, MD, PhD', Zeynep H.C. Akdemir, PhD', \\ Shalini N. Jhangiani, $\mathrm{MS}^{3}$, Marjorie A. Withers, BS', \\ Meryem Tuba Goksungur, MD ${ }^{4}$, Claudia M.B. Carvalho, PhD ${ }^{1}$, Dirk Czesnik, MD ${ }^{5}$, \\ Claudia Gonzaga-Jauregui, PhD', Wojciech Wiszniewski, MD, PhD', \\ Donna M. Muzny, MS ${ }^{3}$, Richard A. Gibbs, PhD ${ }^{3}$, Bernd Rautenstrauss, $\mathrm{PhD}^{6,10}$, \\ Michael W. Sereda, MD ${ }^{5,7}$, James R. Lupski, MD, PhD, DSc(hon) $)^{1,3,8,9}$
}

\begin{abstract}
Purpose: Charcot-Marie-Tooth (CMT) disease is a heterogeneous group of genetic disorders of the peripheral nervous system. Copy-number variants (CNVs) contribute significantly to CMT, as duplication of PMP22 underlies the majority of CMT1 cases. We hypothesized that $\mathrm{CNVs}$ and/or single-nucleotide variants (SNVs) might exist in patients with CMT with an unknown molecular genetic etiology.

Methods: Two hundred patients with CMT, negative for both SNV mutations in several CMT genes and for CNVs involving PMP22, were screened for CNVs by high-resolution oligonucleotide array comparative genomic hybridization. Whole-exome sequencing was conducted on individuals with rare, potentially pathogenic CNVs.
\end{abstract}

Results: Putatively causative CNVs were identified in five subjects ( 2.5\%); four of the five map to known neuropathy genes. Breakpoint sequencing revealed $\mathrm{Alu}$-Alu-mediated junctions as a predominant contributor. Exome sequencing identified MFN2 SNVs in two of the individuals.

Conclusion: Neuropathy-associated CNV outside of the PMP22 locus is rare in CMT. Nevertheless, there is potential clinical utility in testing for CNVs and exome sequencing in CMT cases negative for the CMT1A duplication. These findings suggest that complex phenotypes including neuropathy can potentially be caused by a combination of SNVs and CNVs affecting more than one diseaseassociated locus and contributing to a mutational burden.

Genet Med advance online publication 17 September 2015

Key Words: array comparative genomic hybridization; Charcot-Marie-Tooth disease; exome sequencing; MPZ; PRICKLE1

\section{INTRODUCTION}

Charcot-Marie-Tooth (CMT) disease is a clinically and genetically heterogeneous group of disorders of the peripheral nervous system, with a prevalence of $\sim 1$ in 2,500, making it among the most common inherited neurologic disorders. CMT is characterized by distal weakness, muscular atrophy, foot deformities, gait disturbance, and loss of tendon reflexes accompanied by a relatively sharply demarcated loss of sensation presenting as a distal symmetric polyneuropathy. ${ }^{1}$ The classification was initially developed according to neuropathological and electrophysiological observations, with CMT type 1 and CMT type 2 representing demyelinating and axonal neuropathies, respectively. Gene discoveries within the past two decades have challenged this simplistic classification of CMT. Approximately 70 genes and 10 additional genomic loci have been linked to variants that can cause a CMT-like peripheral neuropathy, making it one of the most genetically diverse neurological phenotypes. All forms of Mendelian inheritance patterns (autosomal dominant, $\mathrm{X}$-linked, and autosomal recessive) have been observed in CMT. ${ }^{2}$

A recurrent 1.4-Mb duplication of a chromosomal segment involving peripheral myelin protein 22 (PMP22) is mediated by nonallelic homologous recombination and is the major molecular genetic subgroup of CMT, accounting for 70-80\% of CMT1 and $~ 50 \%$ of all CMT cases (OMIM 118220). ${ }^{3,4}$ The reciprocal $1.4-\mathrm{Mb}$ deletion underlies the more clinically mild hereditary neuropathy with liability to pressure palsies (OMIM 162500). ${ }^{5}$ Diagnostic testing identified a PMP22 duplication or deletion in $>78 \%$ of $\sim 17,000$ patients with clinical neuropathy for whom a molecular diagnosis was concluded. Either

\footnotetext{
The first two authors contributed equally to this work and are co-first authors.

${ }^{1}$ Department of Molecular and Human Genetics, Baylor College of Medicine, Houston, Texas, USA; ${ }^{2}$ Section of Pediatric Neurology, Texas Children's Hospital, Houston, Texas, USA; ${ }^{3}$ Human Genome Sequencing Center, Baylor College of Medicine, Houston, Texas, USA; ${ }^{4}$ Department of Neurology, Istanbul University, Istanbul Medical Faculty, Istanbul, Turkey; Department of Clinical Neurophysiology, University Medical Center Göttingen, Göttingen, Germany; ${ }^{6}$ Medizinisch Genetisches Zentrum, Munich, Germany; ${ }^{7}$ Max-Planck Institute of Experimental Medicine, Göttingen, Germany; ${ }^{8}$ Department of Pediatrics, Baylor College of Medicine, Houston, Texas, USA; ${ }^{9}$ Texas Children's Hospital, Houston, Texas, USA; ${ }^{10}$ Deceased; Obituary-Neuromuscular Disorders 2015;25:725-726.. Correspondence: James R. Lupski (jlupski@bcm.edu)
} 
copy-number variants (CNVs) or single-nucleotide variants (SNVs) involving four genes-PMP22, GJB1 (Cx32), MPZ, or MFN2-accounted for $~ 95 \%$ of disease-causative mutations. ${ }^{6}$

We previously reported nonrecurrent, rare, unique CNVs involving the PMP22 region in patients with CMT1A or hereditary neuropathy with liability to pressure palsies. ${ }^{7}$ Underlying molecular mechanisms for rare, nonrecurrent PMP22 CNVs differ from the recurrent events mediated by nonallelic low copy repeats acting as ectopic homologous recombination substrates. ${ }^{8}$ These nonrecurrent rearrangements are potentially mediated by replicative mechanisms such as fork-stalling, template switching, microhomology-mediated, break-induced replication, ${ }^{9,10} A l u$-Alu-mediated recombination events, ${ }^{11,12}$ and nonhomologous end joining. CNVs involving CMT genes other than PMP22 have rarely been identified. We previously reported three different deletion CNVs in Cx32 in patients with $\mathrm{X}$-linked CMT; breakpoint junction studies showed either nonhomologous end joining or Alu-Alu-mediated events underlying CNV formation. ${ }^{13}$ We also independently described a homozygous intragenic duplication $\mathrm{CNV}$ in NDRG1 leading to autosomal recessive CMT4D in our current CMT cohort. ${ }^{14}$ In addition, two different CNVs involving amplification of $M P Z$ have been identified..$^{15,16}$

With recent advances in technologies such as array comparative genomic hybridization (aCGH) and genomic sequencing, it is now appreciated that CNVs may encompass more nucleotide variation than SNVs when comparing a given genome with the haploid reference. ${ }^{17}$ We investigated the frequency of CNVs involving non-PMP22 CMT genes through the study of a large cohort of subjects with molecularly undefined CMT. We also sought to identify possible novel candidate genes by examining linkage regions. Finally, we investigated the hypothesis that a combination of $\mathrm{CNV}$ and a rare $\mathrm{SNV}$ mutation can contribute to patient phenotypes by performing whole-exome sequencing (WES) in the subjects in whom a candidate CMT-associated $\mathrm{CNV}$ was identified. Five of 200 individuals with phenotypically characterized CMT that was molecularly undefined contained CNVs in genes potentially responsible for their neuropathy, generating an overall rate of $\sim 2.5 \%$. One of these genes was previously unlinked (KIF24) to peripheral neuropathy, and another had been associated with seizures and a sensory neuropathy, but not a motor neuropathy (PRICKLE1). WES elucidated MFN2 variants in two individuals in whom a CNV in a CMT gene was found. These findings suggest the possibility that either SNV or CNV mutations in a CMT gene, and potentially a combination of both SNVs and CNVs in multiple CMT genes through a mutational burden mechanism, ${ }^{18}$ may contribute to complex neurological phenotypes.

\section{MATERIALS AND METHODS}

\section{Subjects}

This study was approved by the institutional review board at Baylor College of Medicine. Two hundred DNA samples from unrelated subjects with CMT were studied. Informed consent was obtained and peripheral blood then was drawn from patients and family members. DNA was isolated either directly from blood or transformed lymphoblastoid cell lines using a Gentra Puregene purification kit (Qiagen, Valencia, CA). Patients were screened previously for PMP22 CNVs. Most patients were screened for point mutations or SNVs in many of the known CMT genes, including PMP22, MPZ, and GJB1.

\section{Array comparative genomic hybridization}

We designed a high-density oligonucleotide aCGH microarray $(8 \times 60 \mathrm{~K}$ format $)$ that covered 67 neuropathy genes $( \pm 10$-kb flanking regions) and 10 known linkage regions (Supplementary Table S1 online) using the Agilent eArray system (http://earray.chem.agilent.com/earray). This targeted aCGH design for investigating CNVs in CMT has an average genomic resolution of $\sim 1$ probe $/ 200 \mathrm{bp}$ for the 67 gene regions and $\sim 1$ probe $/ 5 \mathrm{~kb}$ for 10 linkage regions. The design did not allow evaluation far upstream of $M P Z$ (in BAB7807 we detected a CNV larger than our array coverage); therefore we designed a specific tiling-path $4 \times 44 \mathrm{~K}$ aCGH spanning $1 \mathrm{Mb}$ to further define the MPZ CNV. Arrays were conducted according to the Agilent oligonucleotide aCGH protocol (version 6.0) and previously described methods. ${ }^{19}$ Gender-matched male (NA10851) and female (NA15510) control DNAs were used for the hybridization procedure (obtained from Coriell Cell Repositories; http://ccr.coriell.org).

\section{Breakpoint junction studies}

Based on aCGH data, we designed primers flanking the boundaries of each deleted/duplicated segment. For duplicated segments, primers were embedded within the duplicated segment in an outward-facing orientation (tandem duplication), and for deleted segments primers were placed outside the deleted segment in an inward-facing direction. Both long-range and conventional polymerase chain reaction (PCR) amplification methods were used to detect the breakpoint junctions.

Primers for patient-specific breakpoint junction amplification are presented in Supplementary Table S2 online. Amplicons from patients BAB392, BAB1219, and BAB7807 were further analyzed by primer walking. We aligned breakpoint sequences manually or using MultAlin (http://multalin.toulouse.inra.fr/ multalin/). ${ }^{20}$

\section{Reverse-transcription PCR and sequencing}

Lymphoblastoid cells from BAB1415 and BAB392 were cultured using standard practices. RNA was extracted using a Qiagen RNeasy Plus miniprep kit. RNA was quantified via NanoDrop, and integrity was determined by agarose electrophoresis.

Reverse transcription (RT) was carried out using qScript cDNA super mix (Quanta Biosciences, Gaithersburg, MD) with $0.5 \mu \mathrm{g}$ of RNA. PCR amplification of RT products was performed by TaKaRa LA taq using the primers listed in Supplementary Table S2 online. Amplification was performed for 32 cycles, and resultant amplicons were sequenced to ensure they were on target. Multiple RNA and complementary DNA reactions were used for each individual; results were consistent across three separate replicates. 


\section{Exome sequencing}

We applied WES to samples BAB7807, BAB1219, BAB392, BAB1415, and BAB3724 at the Baylor College of Medicine Human Genome Sequencing Center through the Baylor-Hopkins Center for Mendelian Genomics research initiative. We used publicly available databases such as the 1000 Genomes Project (http://www.1000genomes.org) and other large-scale exome sequencing projects, including the Exome variant server for the National Heart, Lung, and Blood Institute's GO Exome Sequencing Project, Seattle, WA (http:// evs.gs.washington.edu/EVS/), and the Atherosclerosis Risk in Communities Study Database (http://drupal.cscc.unc.edu/ aric/) as well as our in-house-generated exomes (from $\sim 4,000$ individuals) at Baylor College of Medicine for analyses of candidate variants/mutations. All experiments and analyses were performed according to previously described methods. ${ }^{21}$ SNVs and insertion/deletion mutations in known neuropathy genes were included in Table 1 if they had both an Exome Sequencing Project minor allele frequency $\leq 1 \%$ and an internal database minor allele frequency $\leq 1 \%$. Sanger sequencing verified all the candidate mutations presented in Table 1.

We then determined whether we could detect potential CNVs from WES using the program CoNIFER. ${ }^{22}$ This program predicts duplications and deletions via differences in WES read depth that deviate from a large number of genomes ascertained using the same exome capture and sequencing methodology.

\section{Clinical information}

\section{RESULTS}

Patient BAB7807 presented at about 23 years of age with progressive weakness, myoclonus, fatigue, gait disturbance, and paraesthesias. Physical examination revealed distal accentuated polyneuropathy with mild atrophy of the hand and foot muscles, absent ankle deep tendon reflex, hypoesthesia of the hands and feet, and reduced heat and cold sensation in the distal extremities. Cranial nerves were grossly intact. Myography revealed no positive sharp waves and showed mild chronic denervations of the right tibialis muscle anterior. Nerve conduction velocities were consistent with demyelinating neuropathy (Table 1). Nerve biopsy was suggestive of a mild chronic degenerative demyelinating neuropathy, with no signs of vasculitis or neuritis. Muscle biopsy showed mild neurogenic changes without signs of myopathy or myositis.

BAB1219 is a 35-year-old woman. She presented in elementary school with ankle weakness and distal lower limb paresthesias, in addition to leg pain/cramping after walking long distances. She also reported mild hand weakness. The patient was one of five siblings, and family history was negative for neuromuscular complaints. Physical examination showed bilateral distal muscle weakness in her lower extremities and symmetrically diminished sensation in both distal upper and lower extremities. Electromyography study showed the presence of severe, generalized distally

Table 1 Motor nerve conduction studies of four patients in the study.

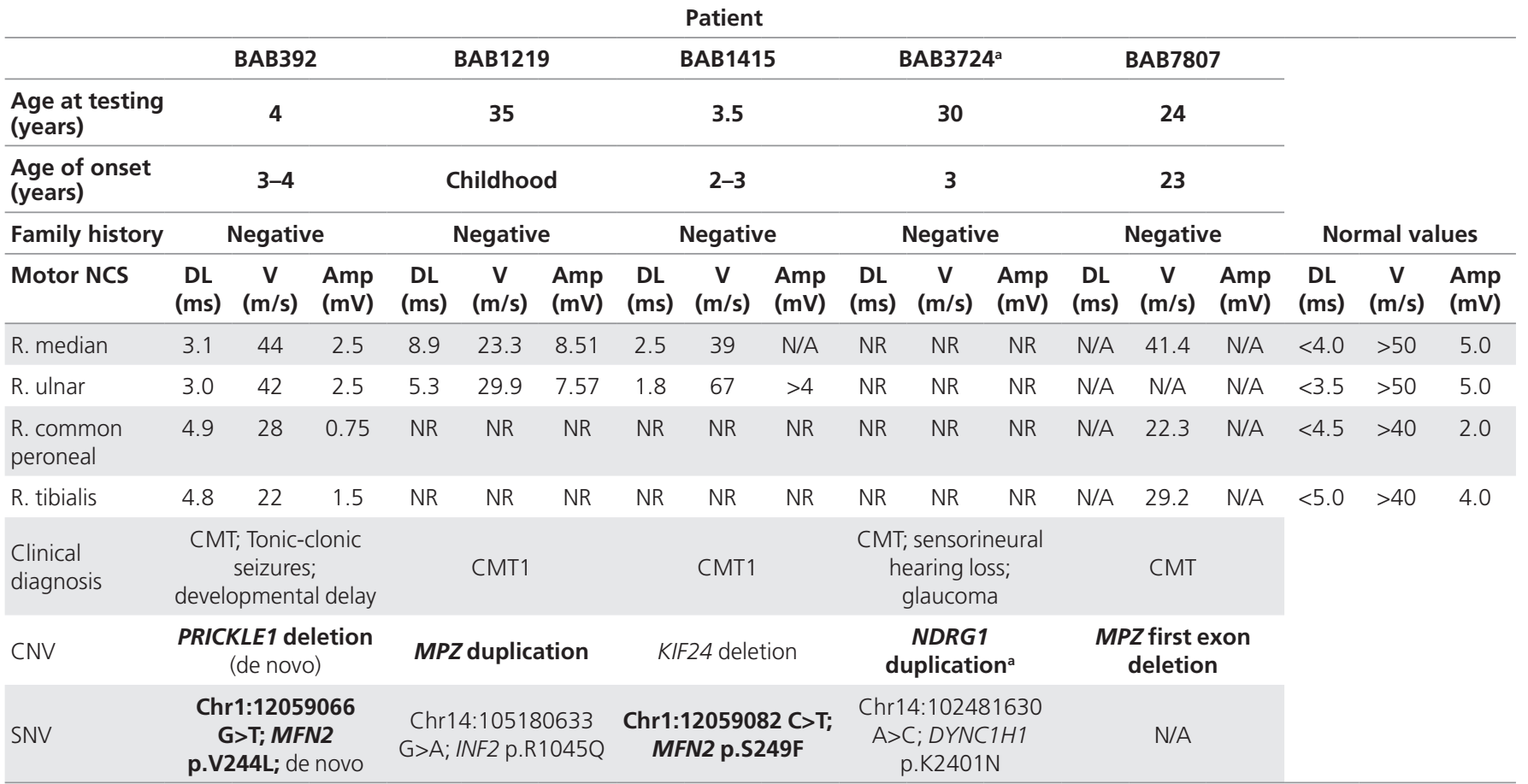

Boldface type indicates likely pathogenic mutations.

Amp, amplitude; CMT, Charcot-Marie-Tooth; CNV, copy-number variant; DL, distal latency; NCS, nerve conduction studies; N/A, not available; NR, not recordable; R., right; SNV, single-nucleotide variant; $V$, conduction velocity.

aPreviously published NDRG1 CNV is homozygous and is a known recessive locus. All others are heterozygous and have known or postulated autosomal dominant mechanisms. 


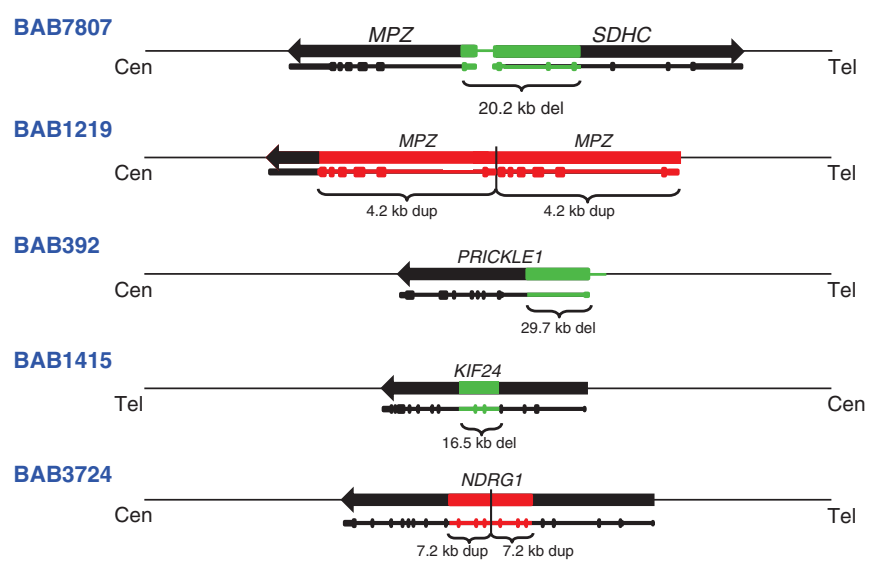

Figure 1 Summary of copy-number variants (CNVs) found in CharcotMarie-Tooth genes and linkage regions. Identification numbers are shown in the left column. Genes are depicted by black horizontal arrows with the arrowhead showing their direction of transcription; cen, centromere; tel, telomere (all genes shown with respect to the + strand). Green boxes depict the position of deletion CNVs, and red boxes depict the position of duplication CNVs. Depiction of the gene structures, including deleted or duplicated exons (vertical bars), is shown below the schematic of the regions. Size of the CNVs is indicated below the colored boxes.

prominent, mixed sensory and motor neuropathy with prominent demyelinating features.

BAB392 was clinically evaluated at 4 years of age because of weakness in his legs. The patient's right foot was pointing inward at birth, and his left foot soon followed. Motor development was consistent with his age; he walked at $\sim 9$ months but tended to fall frequently. Generalized tonic-clonic seizures developed between the ages of 7 and 13 years and were treated with phenobarbital. He also had mild intellectual disability. Follow-up evaluation at 17 years of age revealed that the patient was wheelchair-bound with prominent muscle atrophy in the hands and distal lower extremities. Deep tendon reflexes were absent throughout. Electromyography studies showed neuropathic changes of the muscles, most marked distally.

BAB1415 had weakness in her legs starting at around 2 years 9 months of age. Hand weakness was progressive and became apparent 2 years later. Physical examination at age 9 years showed bilateral claw hands. Muscle strength was $4 / 5$ in the upper and lower extremity muscle groups. Deep tendon reflexes were normal and symmetric in the upper extremities and absent in the lower extremities. Sensation to light touch was mildly decreased in the distal lower extremities. Gait was abnormal and characterized by bilateral foot drop. Muscle biopsy at 3.5 years was consistent with a chronic demyelinating neuropathy.

Nerve conduction velocity study results for all five patients in this study were conducted (Table 1). Study results for patient BAB3724 have been published. ${ }^{14}$

\section{High-resolution aCGH analyses}

To examine for potential disease-associated $\mathrm{CNV}$ in non-CMT1A patients, we performed whole-genome-targeted aCGH (see Materials and Methods) in 200 families with
CMT who were known to be negative for the most common CMT-causative mutations. Our approach revealed five novel CNVs (in four known CMT genes and one candidate CMT gene) ranging in size from 4.2 to $30 \mathrm{~kb}$ (Figure 1). An intragenic homozygous exon 6 to 8 duplication in NDRG1 was previously published in a family with recessive CMT. ${ }^{14}$ We now report the characterization of CNVs in the remaining four subjects with neuropathy.

In two subjects we detected CNVs of $M P Z$ consisting of a 20.2-kb deletion and a 4.2-kb duplication. Subject BAB7807 had a 20.2-kb deletion involving the first coding exon of $M P Z$ and the first three exons of the SDHC gene (Figure 2a,c). Our specific aCGH design allowed us to evaluate the upstream deletion region and to detect the breakpoint junction interval (Figure 2b). Subject BAB1219 had a 4.2-kb duplication involving all coding exons, the $5^{\prime}$ untranslated region and a portion of the $3^{\prime}$ untranslated region of $M P Z$ (Figure 2d,f). This MPZ duplication was independently confirmed by multiplex ligation-dependent probe amplification (data not shown). Patient BAB392 had an intragenic 29.7-kb deletion involving the first exon of PRICKLE1 (RefSeq gene ID NM_153026.2) (Figure 3a,e). Individual BAB1415 has a 16.5-kb deletion involving exons 5 and 6 of KIF24 (RefSeq gene ID NM_194313.2) (Figure 4a,d).

\section{Breakpoint junction sequencing}

Breakpoint junction sequencing was performed in all cases to fine-map CNVs and to gain potential insights into the underlying mechanisms for rearrangement formation. PCR amplification of junctions was achieved for all five CNVs. Patient BAB7807 harbors a deletion with Alu elements at both proximal and distal breakpoints (FLAM_C within intron one of $M P Z$ and AluSz6-D at distal junction). Although the lengths of the repeats differ (137bp for FLAM_C and 312 bp for the AluSz6-D), the two short interspersed elements share $23 \mathrm{bp}$ of perfect sequence identity at the breakpoint junction (Figure $2 b$ ). The two short interspersed elements are highly divergent, yet the junction creates a chimeric FLAM/AluS element.

BAB1219 contained a 4.2-kb tandem MPZ duplication involving all coding regions of the gene and the $5^{\prime}$ untranslated region; the distal breakpoint lies $10 \mathrm{bp}$ upstream of the transcription start site. Breakpoint analysis revealed a 2-bp microhomology (CC) at the junction, consistent with a microhomology-mediated mechanism such as fork stalling template switching underlying this duplication. ${ }^{10}$ No repetitive sequence was observed at either the proximal or the distal breakpoint junctions (Figure 2e).

Breakpoint amplification of BAB392 also revealed short interspersed elements at both proximal and distal regions of the deletion (AluSp is proximal and 304 bp in length, and AluSx is distal and $310 \mathrm{bp}$ in length). The Alu elements shared $16 \mathrm{bp}$ of microhomology, whereas overall they share only $78 \%$ sequence identity (Figure 3b). This junction creates a chimeric Alu element between the two AluS elements. As parental samples were available for $\mathrm{BAB} 392$, we also were able to determine that this deletion is de novo in the proband (Figure 3c). 
a

BAB7807 1923.3

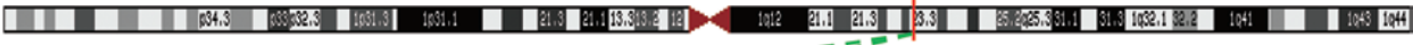

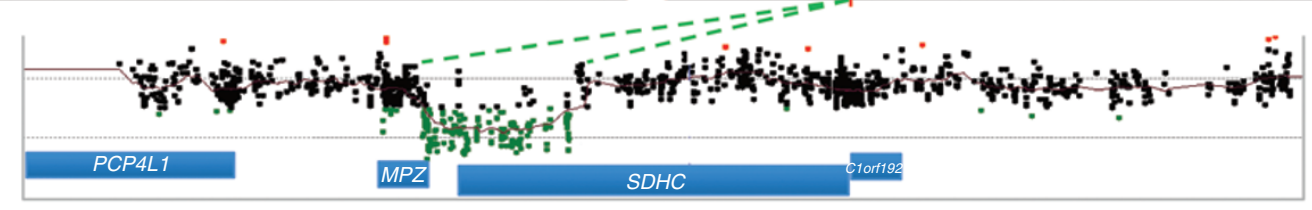

b

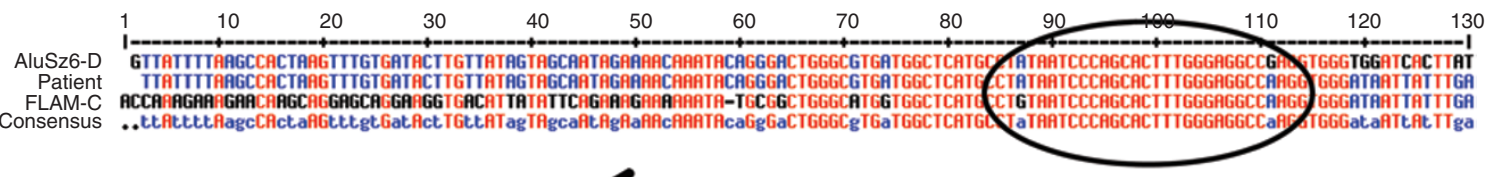

C

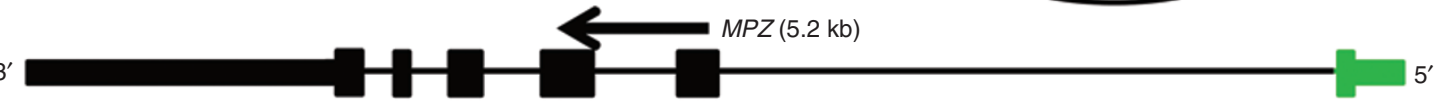

d $\mathrm{BAB} 1219$

$1 \mathrm{q} 23.3$

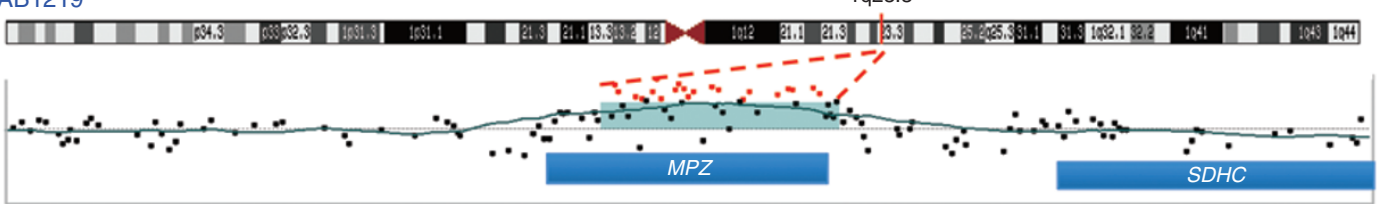

e

Proximal CTCTGCTCATCCTTTCGTAGCTCCATCTCGATGACCATCACCTTTGGGCCTTTGGCGGACTCC

Patient AGAAAGTGGGGGACCAGGAACTGAACGGGGGGTTCCATCACCTTTGGGCCTTTGGCGGACTCC

Distal AGAAAGTGGGGGACCAGGAACTGAACGGGGGGTTCCTGGAACCTGCTTAAAATCCCCTAGGGC

f

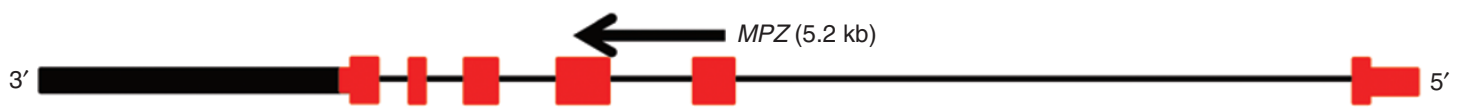

Figure 2 Array comparative genomic hybridization (CGH) and breakpoint junction studies of the MPZ gene deletion (in BAB7807) and duplication (in BAB1219). (a) Chromosome 1 ideogram with G bands indicated (top). The location of MPZ is shown with a red vertical line at 1q23.3. Array CGH results of the 20-kb deletion involving upstream parts of both MPZ and SDHC. (b) Breakpoint sequence analysis for MPZ deletion using the MultAlin alignment tool. Proximal and distal reference sequences are mapped within FLAM_C and AluSz6-D sequences, respectively. The patient's breakpoint sequence that matches the reference sequence is shown in blue, mismatched sequence is shown in black, and perfect matching between all three sequences are shown in red. The breakpoint junction where the transition occurred from the proximal to the distal reference sequence is specified with a black circle. (c) Graphic representation of MPZ (vertical bars; deleted exon 1 is shown in green); size and orientation of the gene are shown above the exons. (d) Chromosome 1 ideogram with $\mathrm{G}$ bands as described in Figure 2a. Array CGH results reveal genomic duplication. The red line indicates MPZ on chromosome 1q23.3. (e) Breakpoint sequence analysis for MPZ duplication. The proximal and distal sequences are from hg19/GRCh37. Proximal reference sequence and patient breakpoint sequences that match the proximal reference sequence are shown in red, the distal reference sequence and patient breakpoint sequences that match the distal reference sequence are shown in green, and 2-bp microhomology (CC) at the junction is shown in purple. (f) Graphical representation of six exons of MPZ (vertical bars; duplicated exons are shown in red); the size and orientation of the gene are shown above the exons.

The deletion of subject BAB1415 also was mediated by two Alu elements, an Alu Ya5 (310 bp) at the proximal region and an $A l u Y(306 \mathrm{bp})$ at the distal region. At the breakpoint junction, the two Alu elements shared $18 \mathrm{bp}$ of perfect sequence identity, yet overall, they share $85 \%$ homology (Figure $4 \mathrm{~b}$ ).

In summary, breakpoint analysis showed that three of the four subjects presented here contained junctions that generate chimeric short interspersed elements. The three Alu-Alu CNVs contained between 16 and $22 \mathrm{bp}$ of microhomology at the junction; the size of the deletions ranged from 16.5 to $29.7 \mathrm{~kb}$. The last patient (BAB1219) contained a duplication that was not mediated by repetitive elements; there were $2 \mathrm{bp}$ of microhomology at the junction.

\section{Aberrant splicing and transcript levels}

For both BAB392 and BAB1415, cultured lymphoblastoid cell lines enabled RNA studies to be performed. In BAB392, the first exon of PRICKLE1 was deleted along with the region directly upstream of the gene. Therefore, we predicted that transcription of PRICKLE1 could be negatively affected by this mutation. RT-PCR amplification of BAB392 RNA suggests decreased levels of PRICKLE1 messenger RNA in relation to both BAB1415 and a control subject without CMT. This difference was seen for two different primer pairs (exon 2-1 forward to 5 reverse and a second exon 2-2 forward primer to 4 reverse) (Figure 3d), was consistent across three different RNA preparations and complementary DNA reactions. GAPDH and TBP amplification showed no change in transcript levels in RNAs from multiple patients and controls (data not shown).

For subject $\mathrm{BAB} 1415$, the $\mathrm{CNV}$ is predicted to alter RNA structure by skipping two KIF24 exons (5 and 6). Independent RT-PCR reactions from this patient showed the predicted pattern, resulting in peaks on peaks at the end of exon 4 and the beginning of exon 7 (Figure 4c). Two control RNAs showed the standard pattern of splicing from exons 4 to 5 and 6 to 7 (data not shown). The skipping of exons 5 and 6 in BAB1415 
<smiles>[R15][R15]</smiles>

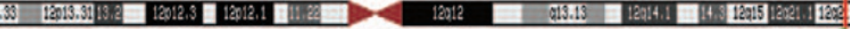

b

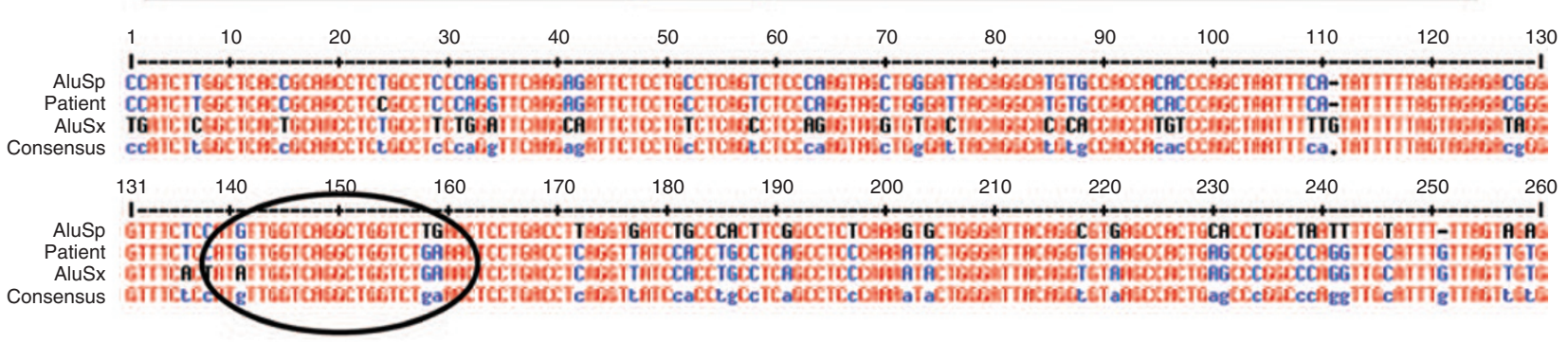

c

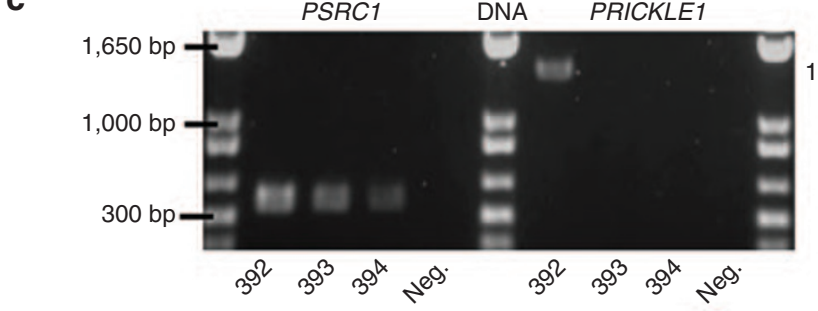

d

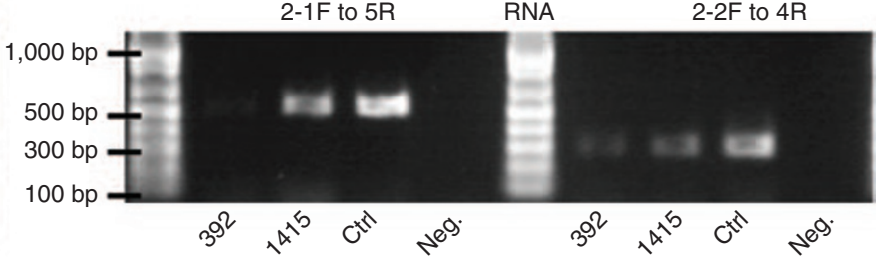

e

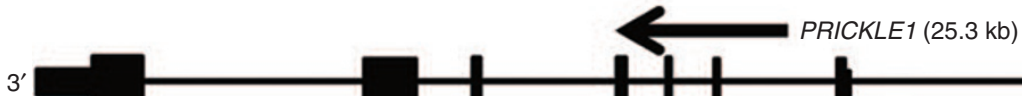
$5^{\prime}$

Figure 3 Array comparative genomic hybridization (CGH) and breakpoint junction studies of the PRICKLE1 deletion in BAB392. (a) Chromosome 12 ideogram with $\mathrm{G}$ bands indicated (top). The location of PRICKLE1 is shown with a red vertical line at $12 \mathrm{q} 12$. Array CGH results of the $\sim 30-\mathrm{kb}$ deletion involving the $5^{\prime}$ untranslated region and start of the PRICKLE1 coding sequence. (b) Breakpoint sequence analysis for the PRICKLE1 deletion using the MultAlin alignment tool. Proximal and distal reference sequences are mapped within AluSp and AluSx sequences, respectively. Sequences matching the consensus Alu sequence are shown in blue, the mismatched sequence is shown in black, and concordance between the three Alu sequences is depicted in red. The breakpoint junction is specified with a black circle where the transition occurred from the proximal to the distal reference sequence. (c) Polymerase chain reaction (PCR) confirmation of the junction present in the BAB392 sample. Amplification of PSRC1 gene serves as a control. Breakpoint amplification is successful in the patient ( 1.5-kb band) and not parental (BAB393 and BAB394). (d) Reverse-transcriptase PCR depicting relatively decreased transcript levels of PRICKLE1 in BAB392 is shown. The amount of amplification from BAB392 is lower than two controls who lack CNV in PRICKLE1. This is consistent with the $5^{\prime}$ untranslated region deletion possibly leading to decreased transcription of the gene or nonsense-mediated decay of the transcript. (e) Graphic view of eight exons of PRICKLE1 (vertical bars, deleted first exon is shown in green); the size and orientation of the gene are noted above the exons.

predicts a messenger RNA that upon translation will terminate one amino acid downstream of exon 4 (Figure 4c).

\section{Exome sequencing analyses}

The axonal neuropathy present in BAB392 could potentially represent a phenotypic expansion of the sensory neuropathy associated with PRICKLE1 heterozygous mutations. Additionally, the KIF24 deletion in BAB1415 may possibly be unrelated to the neuropathy present in the patient. Because of the uncertainty of genotype-phenotype correlations in these two patients, we conducted WES on all five patients with rare CNVs in CMT genes or linkage regions. With these data, we sought to determine SNV or insertion/deletion mutations that might contribute to the phenotypes of the patients. Sequencing resulted in the discovery of Mitofusin 2, or MFN2, mutations in two patients. The mutations result in an NM_001127660 p.V244L (Chr1:12059066 G>T) and p.S249F (Chr1:12059082 $\mathrm{C}>\mathrm{T}$ ) change in BAB392 and BAB1415, respectively (Table 1).
Alterations of these two amino acid residues are reported to represent causative pathogenic mutations in the Human Gene Mutation Database (http://www.hgmd.cf.ac.uk); however, the mutation previously reported at p.V244 was a G>A transition resulting in a methionine residue. This $\mathrm{V} 244 \mathrm{M}$ mutation is present twice in our in-house database $(\sim 1 / 2,400)$, both in patients with CMT. ${ }^{23}$ The V244L change in BAB392 is also likely to be pathogenic, because the amino acid residue is highly conserved and the mutation is de novo and predicted to be deleterious by various bioinformatic algorithms (Supplementary Figure S1 online). Two of the three other patients also contained a nonsynonymous neuropathy-associated SNV with minor allele frequency $<1 \%$; however, the phenotypes present in BAB1219, BAB3724, and BAB7807 seem to be consistent with gene dosage changes caused by the CNVs present in their genomes. Nevertheless, a contribution of these SNV to a potential mutational burden cannot be excluded. ${ }^{18}$ Parental samples were available only for BAB392 and BAB3724. 
a BAB1415 9p13.3

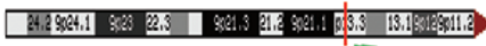

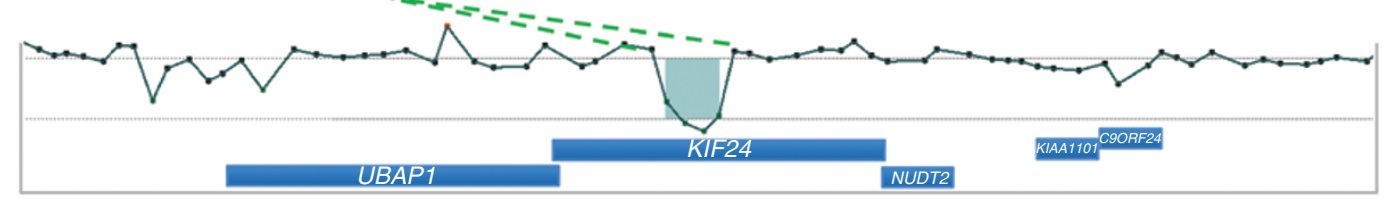

b

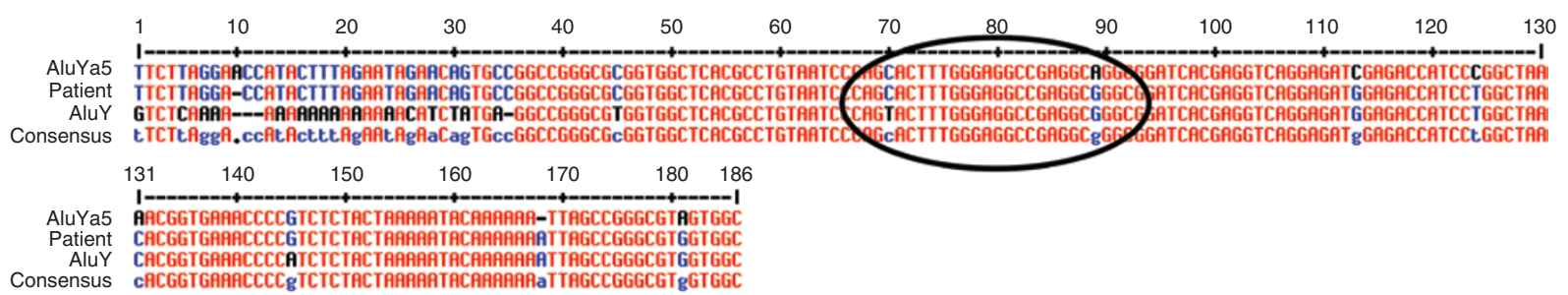

C

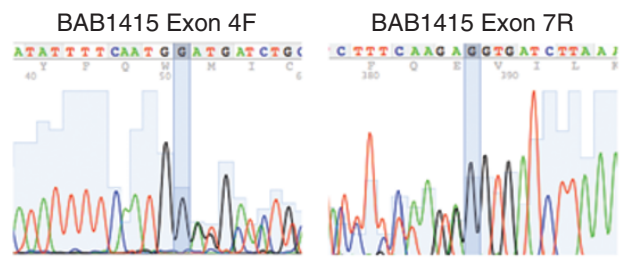

Exon 4 to exon 7 spliced

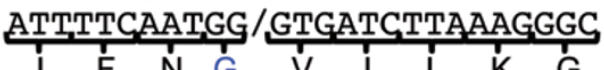

ATTTTCAATGGGTGATCTTAAAGGGC

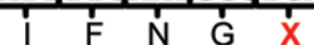

d

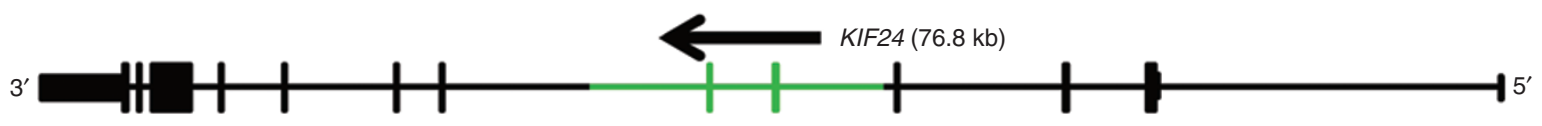

Figure 4 Array comparative genomic hybridization (CGH) and breakpoint junction studies of the KIF24 gene deletion in BAB1415. (a) Chromosome 9 ideogram with G bands indicated (top). The location of KIF24 is shown with a red vertical line at 9p13.3. Array CGH results of the $\sim 16.5-\mathrm{kb}$ deletion involving exons 5 and 6 of KIF24. (b) Breakpoint sequence for KIF24 deletion using the MultAlin alignment tool. Proximal and distal reference sequences are mapped within AluYa5 and AluY sequences, respectively. The patient's breakpoint sequence that matches the reference sequence is shown in blue, the mismatched sequence is shown in black, and perfect matching between all three sequences is depicted in red. The breakpoint junction is specified with a black circle where the transition occurred from the proximal to the distal reference sequence. (c) Skipping of exons 5 and 6 because of the deletion is confirmed in the complementary DNA. Peaks on peaks at the end of exon 4 and the beginning of exon 7 show the two species of RNA in BAB1415, both wild-type and deletioncontaining. The lack of exons 5 and 6 in the deletion-containing RNA lead to a premature termination codon at the beginning of exon 7. (d) Graphic view of 13 exons of KIF24 (vertical bars, deleted exons 5 and 6 are shown in green); the size and orientation of the gene are shown above the exons.

Utilizing the WES data, we then determined whether sequencing could predict the CNVs in the five patients. The software CoNIFER ${ }^{22}$ was applied to the WES data from the patients; however, none of the experimentally discovered and verified CNVs was bioinformatically predicted from WES data.

\section{DISCUSSION}

Our aCGH approach detected nonrecurrent CNVs in $M P Z$ (two), PRICKLE1, KIF24, and NDRG1 (ref. 14) in 200 molecularly unresolved CMT cases. Deletion and duplication sizes ranged from 4 to $30 \mathrm{~kb}$ (Figure 1), encompassing anywhere from one exon to the entire gene. Breakpoint junction studies revealed that Alu-Alu-mediated rearrangements (present in three of five junctions) are the predominant molecular mechanism in CNVs present in CMT cases negative for CMT1A duplication.

Zhang et al. ${ }^{24}$ analyzed nonrecurrent CNVs in the $17 \mathrm{p} 12$ region and found that mechanisms other than nonallelic homologous recombination, for example, fork-stalling, template-switching, microhomology-mediated, break-induced replication, nonhomologous end joining, and Alu-mediated recombination events, contributed to nonrecurrent $\mathrm{CNV}$ formation in CMT1A. Recently, 57 deletion and duplication breakpoints at the SPAST locus were examined and $\sim 70 \%$ of these events involved directly-oriented Alu elements, illustrating the predominance of $\mathrm{Alu}$-mediated mechanisms in nonrecurrent rearrangements. ${ }^{11}$ Early studies showed two of four nonrecurrent CNVs associated with Smith-Magenis syndrome were due to Alu-mediated mechanisms. ${ }^{25}$ Additionally, a recent study showed that $\sim 80 \%$ of simple deletions and $\sim 50 \%$ of tandem duplications at $17 \mathrm{p} 13.3$ are mediated by Alu-Alu events. ${ }^{12}$ These data show that the Alu-Alu-mediated mutational mechanism is prevalent in nonrecurrent rearrangements and is responsible for over $50 \%$ of the CNVs presented here.

Our approach reveals that 4 of 200 individuals (2\%) have a $\mathrm{CNV}$ in a known neuropathy gene (MPZ (two individuals), PRICKLE1, and NDRG1). However, the sizes of the CNVs detected herein may be too small to identify by some currently used commercial molecular diagnostic techniques. Methods including locus-specific tests such as fluorescence in situ hybridization (FISH) or genome-wide assays such as SNP arrays may 
be insufficient, and high-resolution aCGH may be required in cases negative for CMT1A duplication or SNV mutations. Huang et al. ${ }^{26}$ performed aCGH targeting 34 hereditary peripheral neuropathy regions in 97 patients who were screened for the most common CMT genes. They found an exonic and an intronic CNV in ARHGEF10, an intronic loss in SPTLC1, and an exonic loss in MTMR2; these were interpreted as polymorphic variants because of their frequency or intronic location. A smaller, nonrecurrent duplication of PMP22 was also identified. Among our 200 samples we found $31 \mathrm{CNV}$ polymorphisms in addition to the five variants reported here (Supplementary Table S3 online). The five disease-associated and candidate $\mathrm{CNV}$ s are not present in the database of genomic variants.

Deletion and duplication of the $M P Z$ gene were found in BAB7807 and BAB1219, respectively. Interestingly, although SNV mutations of this gene are common, CNVs involving $M P Z$ are not prevalent in CMT. Mutations in MPZ are associated with CMT1B, an autosomal dominant form of the disease. ${ }^{27}$ The deletion of the first exon of MPZ in BAB7807 continues to contribute to the range of mutations that lead to perturbation of $M P Z$ expression.

Duplication of all coding exons of $M P Z$ was recently reported..$^{15}$ The duplication cosegregated with CMT in 12 tested individuals; the coordinates presented indicate that the BAB1219 mutation is probably different and therefore a novel MPZ duplication. Additionally, a three-generation family with a quadruplication of MPZ and neighboring SDHC and C1orf192 genes (six members affected and four unaffected) was recently reported. ${ }^{16}$ Those authors further demonstrated the increased expression of $M P Z$ in lymphoblastoid cell lines ( $5 \times$ wild-type) and sural nerves $(3 \times)$ in the affected individuals. The phenotype of BAB1219 is consistent with previous clinical findings of $M P Z$ duplication cases, which have a demyelinating sensorimotor neuropathy phenotype that starts during childhood. As the number of copies of $M P Z$ increases, myelination of nerve fibers decreases gradually. ${ }^{28}$ These data support the contention that $M P Z$, like $P M P 22$, is dosage sensitive, and that rearrangement leading to either increased or decreased copy number can cause peripheral neuropathy. Mutations (point mutations and copy-number gains) in $M P Z$ are associated with a broad phenotypic spectrum, ranging from severe onset during the neonatal period to late onset of milder symptoms resulting from hypomyelination..$^{15}$ The $M P Z$ duplication in BAB1219 seems to be more deleterious than the deletion present in BAB7807 in terms of both age at onset and nerve conduction velocity (Table 1). This seems consistent with the previously reported observation that haploinsufficiency of $M P Z$, generated by nonsense-mediated decay of a truncated transcript, is associated with a less severe phenotype than aggregation of MPZ protein within the endoplasmic reticulum. ${ }^{29,30}$

Patient BAB392 had a deletion of the $5^{\prime}$ untranslated region of PRICKLE1. Homozygous mutations in PRICKLE1 (OMIM 608500) were first described in three consanguineous families with progressive myoclonus epilepsy-ataxia syndrome; one family presented with a mild neuropathy (OMIM 612437). ${ }^{31}$ Heterozygous missense mutations of both PRICKLE1 and PRICKLE2 and deletions of PRICKLE2 were found in patients with myoclonic/generalized tonic-clonic seizure responsive to valproic acid and/or developmental delay in humans, mice, and flies. ${ }^{32}$ Our patient had similar findings, including generalized tonic-clonic seizures that responded to treatment with phenobarbital (valproic acid and phenobarbital have antiepileptic effects through $\gamma$-aminobutyric acid receptors). Bosoi et al. ${ }^{33}$ recently reported heterozygous variants in PRICKLE1 in human subjects with neural tube defects.

We also found a two-exon deletion CNV in KIF24 in BAB1415. Furthermore, RNA sequencing showed heterozygous exon 5 and 6 skipping in the affected individual (Figure 4). KIF24 is a member of the kinesin gene family and is a ubiquitously expressed gene associated with microtubules. No mutation has been reported as causative of diseases in KIF24. Mutations in other members of the kinesin family have been associated with peripheral neuropathies, including KIF1B and CMT type 2A1 (OMIM 118210) and KIF5A and spastic paraplegia with peripheral neuropathy. ${ }^{34,35} \mathrm{~A}$ lossof-function mutation in KIF1B was identified in a family with autosomal dominant CMT2 family; however, mutations in MFN2 are the primary pathologic segregating mutation in CMT2A..$^{34,36}$

WES was conducted to determine whether KIF24 was a likely CMT candidate gene in BAB1415 as well as the severity of the neuropathy in BAB392. This led to the discovery of MFN2 mutations in the two individuals. The likely molecular diagnosis in BAB1415 is a previously reported MFN2 mutation, and the contribution of the KIF24 CNV remains to be determined. By contrast, BAB392 presented with a composite phenotype consisting of seizures and neuropathy. It is likely that the PRICKLE1 CNV combined with the MFN2 SNV led to the patient's manifestations. Interestingly, when parental samples were examined, both the PRICKLE1 deletion CNV and the MFN2 SNV were de novo in the patient. This depicts the necessity of considering all possible rationales for a patient phenotype, even with a likely causative new mutation. Blended phenotypes have been observed previously with two SNV point mutations in an individual, comprising $~ 5 \%$ of diagnostic dilemmas referred for potential molecular diagnosis by exome sequencing. ${ }^{37,38}$

In summary, CNVs are a relevant cause of CMT in patients with unknown molecular genetic etiology (responsible for $2 \%$ of patients without CMT1A), but such variants are rare in genes other than PMP22. Our approach allowed detection of, to our knowledge, the first disease-associated autosomal recessive duplication $\mathrm{CNV}$ in a rare neuropathy gene, NDRG1 (ref 14), as well as an $M P Z$ deletion associated with neuropathy. Furthermore, WES combined with $\mathrm{CNV}$ analysis was critical in unraveling the etiology of complex phenotypic presentations and composite neurological phenotypes potentially representing a mutational burden involving more than one gene, and it helped to define candidate mutations.

\section{SUPPLEMENTARY MATERIAL}

Supplementary material is linked to the online version of the paper at http://www.nature.com/gim

\section{ACKNOWLEDGMENTS}

The authors thank the patients and families for their contribution to this study. This work was supported by US National Human Genome Research Institute (NHGRI)/National Heart, Lung, and Blood Institute 
(NHLBI) grant HG006542 to the Baylor-Hopkins Center for Mendelian Genomics, National Institute of Neurological Disorders and Stroke (NINDS) grant NS058529, National Institute of General Medical Sciences (NIGMS) grant GM106373, National Institute of Child Health and Development grant HD083092, and the Intellectual and Developmental Disabilities Research Center. Additional funding was provided by the NHGRI (award U54HG003273 to R.A.G. for the BCM Human Genome Sequencing Center). C.R.B. is a Howard Hughes Medical Institute fellow of the Damon Runyon Cancer Research Foundation (DRG 2155-13). W.W. is supported by NINDS grant K23NS078056. T.H. is supported by the Medical Genetics Research Fellowship Program (NIH/NIGMS NIH T32 GM07526). M.W.S. holds a DFG Heisenberg Professorship (SE 1944/1-1).

\section{DISCLOSURE}

J.R.L. has stock ownership in 23andMe, is a paid consultant for Regeneron Pharmaceuticals, has stock options in Lasergen, Inc., is a member of the Scientific Advisory Board of Baylor Miraca Genetics Laboratories, and is a coinventor on US and European patents related to molecular diagnostics for inherited neuropathies, eye diseases, and bacterial genomic fingerprinting. The Department of Molecular and Human Genetics at Baylor College of Medicine derives revenue from the chromosomal microarray analysis and clinical exome sequencing offered in the Baylor Miraca Genetics Laboratory (http://www.bmgl.com/BMGL/Default.aspx). The other authors declare no conflict of interest.

\section{REFERENCES}

1. England JD, Gronseth GS, Franklin G, et al.; American Academy of Neurology. Practice Parameter: evaluation of distal symmetric polyneuropathy: role of autonomic testing, nerve biopsy, and skin biopsy (an evidence-based review). Report of the American Academy of Neurology, American Association of Neuromuscular and Electrodiagnostic Medicine, and American Academy of Physical Medicine and Rehabilitation. Neurology 2009;72:177-184.

2. Rossor AM, Polke JM, Houlden H, Reilly MM. Clinical implications of genetic advances in Charcot-Marie-Tooth disease. Nat Rev Neurol 2013;9:562-571.

3. Szigeti K, Lupski JR. Charcot-Marie-Tooth disease. Eur J Hum Genet 2009;17:703-710.

4. Lupski JR, de Oca-Luna RM, Slaugenhaupt S, et al. DNA duplication associated with Charcot-Marie-Tooth disease type 1A. Cell 1991;66:219-232.

5. Chance PF, Alderson MK, Leppig KA, et al. DNA deletion associated with hereditary neuropathy with liability to pressure palsies. Cell 1993;72:143-151.

6. DiVincenzo C, Elzinga CD, Medeiros AC, et al. The allelic spectrum of CharcotMarie-Tooth disease in over 17,000 individuals with neuropathy. Mol Genet Genomic Med 2014;2:522-529.

7. Zhang F, Seeman P, Liu P, et al. Mechanisms for nonrecurrent genomic rearrangements associated with CMT1A or HNPP: rare CNVs as a cause for missing heritability. Am J Hum Genet 2010;86:892-903.

8. Liu P, Lacaria M, Zhang F, Withers M, Hastings PJ, Lupski JR. Frequency of nonallelic homologous recombination is correlated with length of homology: evidence that ectopic synapsis precedes ectopic crossing-over. Am J Hum Genet 2011;89:580-588.

9. Hastings PJ, Ira G, Lupski JR. A microhomology-mediated break-induced replication model for the origin of human copy number variation. PLoS Genet 2009; 5:e1000327.

10. Lee JA, Carvalho CM, Lupski JR. A DNA replication mechanism for generating nonrecurrent rearrangements associated with genomic disorders. Cell 2007; 131:1235-1247.

11. Boone PM, Yuan B, Campbell IM, et al. The Alu-rich genomic architecture of SPAST predisposes to diverse and functionally distinct disease-associated CNV alleles. Am J Hum Genet 2014;95:143-161.

12. GuS, Yuan B, Campbell IM, et al. Alu-mediated diverse and complex pathogenic copy-number variants within human chromosome 17 at p13.3. Hum Mol Genet 2015;24:4061-4077.
13. Gonzaga-Jauregui C, Zhang F, Towne CF, Batish SD, Lupski JR. GJB1/Connexin 32 whole gene deletions in patients with $X$-linked Charcot-Marie-Tooth disease. Neurogenetics 2010;11:465-470.

14. Okamoto Y, Goksungur MT, Pehlivan D, et al. Exonic duplication CNV of NDRG1 associated with autosomal-recessive HMSN-Lom/CMT4D. Genet Med 2014;16:386-394.

15. Høyer H, Braathen GJ, Eek AK, Skjelbred CF, Russell MB. Charcot-Marie-Tooth caused by a copy number variation in myelin protein zero. Eur J Med Genet 2011;54:e580-e583.

16. Maeda MH, Mitsui J, Soong BW, et al. Increased gene dosage of myelin protein zero causes Charcot-Marie-Tooth disease. Ann Neurol 2012;71:84-92.

17. Gonzaga-Jauregui C, Lupski JR, Gibbs RA. Human genome sequencing in health and disease. Annu Rev Med 2012;63:35-61.

18. Gonzaga-Jauregui $C$, Harel T, Gambin T, et al. Exome sequence analysis suggests that genetic burden contributes to phenotypic variability and complex neuropathy. Cell Reports 2015; 12:1169-1183.

19. Pehlivan D, Hullings $M$, Carvalho CM, et al. NIPBL rearrangements in Cornelia de Lange syndrome: evidence for replicative mechanism and genotype-phenotype correlation. Genet Med 2012;14:313-322.

20. Corpet F. Multiple sequence alignment with hierarchical clustering. Nucleic Acids Res 1988;16:10881-10890.

21. Yuan B, Pehlivan D, Karaca E, et al. Global transcriptional disturbances underlie Cornelia de Lange syndrome and related phenotypes. J Clin Invest 2015;125:636-651.

22. Krumm N, Sudmant PH, Ko A, et al.; NHLBI Exome Sequencing Project. Copy number variation detection and genotyping from exome sequence data. Genome Res 2012;22:1525-1532.

23. Kijima K, Numakura C, Izumino H, et al. Mitochondrial GTPase mitofusin 2 mutation in Charcot-Marie-Tooth neuropathy type 2A. Hum Genet 2005;116:23-27.

24. Zhang F, Khajavi M, Connolly AM, Towne CF, Batish SD, Lupski JR. The DNA replication FoSTeS/MMBIR mechanism can generate genomic, genic and exonic complex rearrangements in humans. Nat Genet 2009;41:849-853.

25. Stankiewicz P, Shaw CJ, Dapper JD, et al. Genome architecture catalyzes nonrecurrent chromosomal rearrangements. Am J Hum Genet 2003;72: 1101-1116.

26. Huang J, Wu X, Montenegro G, et al. Copy number variations are a rare cause of non-CMT1A Charcot-Marie-Tooth disease. J Neurol 2010;257: 735-741.

27. Hayasaka K, Himoro M, Sato W, et al. Charcot-Marie-Tooth neuropathy type $1 \mathrm{~B}$ is associated with mutations of the myelin P0 gene. Nat Genet 1993;5: 31-34.

28. Wrabetz L, Feltri ML, Quattrini A, et al. $P(0)$ glycoprotein overexpression causes congenital hypomyelination of peripheral nerves. J Cell Bio/ 2000;148: 1021-1034.

29. Inoue K, Khajavi M, Ohyama T, et al. Molecular mechanism for distinct neurological phenotypes conveyed by allelic truncating mutations. Nat Genet 2004;36:361-369.

30. Khajavi M, Inoue K, Wiszniewski W, Ohyama T, Snipes GJ, Lupski JR. Curcumin treatment abrogates endoplasmic reticulum retention and aggregation-induced apoptosis associated with neuropathy-causing myelin protein zero-truncating mutants. Am J Hum Genet 2005;77:841-850.

31. Bassuk AG, Wallace RH, Buhr A, et al. A homozygous mutation in human PRICKLE1 causes an autosomal-recessive progressive myoclonus epilepsy-ataxia syndrome. Am J Hum Genet 2008;83:572-581.

32. Tao $H$, Manak JR, Sowers $L$, et al. Mutations in prickle orthologs cause seizures in flies, mice, and humans. Am J Hum Genet 2011;88:138-149.

33. Bosoi CM, Capra V, Allache R, et al. Identification and characterization of novel rare mutations in the planar cell polarity gene PRICKLE1 in human neural tube defects. Hum Mutat 2011;32:1371-1375.

34. Zhao C, Takita J, Tanaka Y, et al. Charcot-Marie-Tooth disease type 2A caused by mutation in a microtubule motor KIF1Bbeta. Cell 2001;105:587-597.

35. Goizet C, Boukhris A, Mundwiller E, et al. Complicated forms of autosomal dominant hereditary spastic paraplegia are frequent in SPG10. Hum Mutat 2009:30:E376-E385.

36. Züchner S, Mersiyanova IV, Muglia M, et al. Mutations in the mitochondrial GTPase mitofusin 2 cause Charcot-Marie-Tooth neuropathy type 2A. Nat Genet 2004;36:449-451.

37. Yang Y, Muzny DM, Xia F, et al. Molecular findings among patients referred for clinical whole-exome sequencing. JAMA 2014;312:1870-1879.

38. Yang Y, Muzny DM, Reid JG, et al. Clinical whole-exome sequencing for the diagnosis of mendelian disorders. N Engl J Med 2013;369:1502-1511. 